\title{
The set gene is a potential oncogene in human colorectal adenocarcinoma and oral squamous cell carcinoma
}

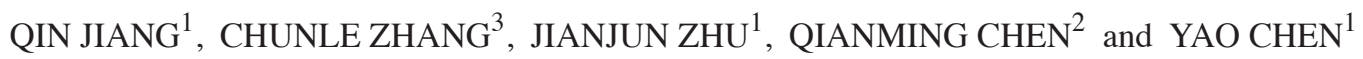 \\ ${ }^{1}$ Department of Anatomy, Premedical and Forensic Medical Institute; ${ }^{2}$ Stomatology Key Laboratory of Disease; \\ ${ }^{3}$ Department of Gastroenterology, West China Hospital, Sichuan University, Chengdu, Sichuan 610041, P.R. China
}

Received February 28, 2011; Accepted June 24, 2011

DOI: $10.3892 / \mathrm{mmr} .2011 .526$

\begin{abstract}
The purpose of this study was to determine whether set gene plays a role in the tumorigenesis of human colorectal adenocarcinoma and oral squamous cell carcinoma. We used the human colon carcinoma cell line Ls174 and oral squamous cell carcinoma cell line HSC3 to evaluate the effect of set suppression on cancer cell proliferation and apoptosis. Using real-time PCR, we examined set gene expression in human colorectal adenocarcinoma tissues and matched normal colorectal tissues. Thirty pairs of colorectal adenocarcinoma tissues and matched normal colorectal tissues were used for real-time PCR. We transfected human colon carcinoma cell line Ls174 and oral squamous cell carcinoma cell line HSC3 with siRNA against the set gene. The effect of set gene suppression on cancer cell proliferation and apoptosis were studied by MTT assay and flow cytometry. Real-time PCR indicated that set gene expression was up-regulated in $70 \%$ of tumor samples (21 out of 30 samples). siRNA2 sequences significantly decreased set mRNA levels in Ls174 and HSC3 cells. The inhibitory rate in the two cell lines was 55.91 and $71.57 \%$, respectively. MTT assay revealed a $21.4 \%$ inhibition on cell proliferation in HS174 cells and a $20.2 \%$ inhibition in HSC 3 cells. Flow cytometry data indicated that the cell apoptosis rate was $18.37 \%$ in Ls174 cells and $17.97 \%$ in HSC3 cells; these rates were significantly higher than those of the control groups. In conclusion, the set gene was found to play a role in the tumorigenesis of human colorectal adenocarcinoma. It may promote tumorigenesis by enhancing cancer cell proliferation and inhibiting cancer cell apoptosis.
\end{abstract}

Correspondence to: Dr Yao Chen, Department of Anatomy, Premedical and Forensic Medical Institute, West China Medical Center, Sichuan University, Chengdu, Sichuan 610041, P.R. China E-mail:xmxfh@263.net

Key words: set, colorectal adenocarcinoma, oral squamous cell carcinoma, real-time PCR, siRNA

\section{Introduction}

Colorectal adenocarcinoma is one of the most common malignant tumors, and its prevalence has been on the increase. Colorectal adenocarcinoma has the second highest incidence among all cancers, and is the second most common cause of cancer-related death worldwide $(1,2)$. On the other hand, the incidence of oral cancer has also exhibited an increase in numerous countries, accounting for $5.6 \%$ of all malignant tumors in China (3). Tumorigenesis is a multi-step process that involves multi-gene change. The investigation of the molecular mechanism of tumorigenesis may aid in improving cancer prevention, early diagnosis, and effective treatment (4).

Using cDNA subtractive library and cDNA microarray techniques, we previously identified a total of $86 \mathrm{cDNA}$ sequences differentially expressed between human colorectal adenocarcinoma tissues and normal colorectal tissues (5). In the present study, we focused on one differentially expressed sequence (Genbank accession number: ES274071), identified its full-length cDNA using BLASTn of NCBI, and identified the set gene (Genbank accession number: NM003011.3). This gene contains 2936 base pairs, with an 834-bp open reading frame. The set gene is located on human chromosome 9p34. It is predicted to encode a 277 amino acid protein with a molecular weight of $39 \mathrm{kDa}$. The set gene has been found to play a role in leukemia and ovarian cancer, but its role in the development of colorectal adenocarcinoma and oral squamous cell carcinoma remains to be elucidated (10-15).

To determine whether the set gene is involved in colorectal adenocarcinoma and oral squamous cell carcinoma, we investigated the set gene expression profile in human colorectal adenocarcinoma and normal colorectal tissues using real-time PCR. RNAi was used to suppress the set gene expression in the human colorectal cancer cell line Ls174 and the human oral squamous cell carcinoma cell line HCS3, and to observe the effect of set gene on cancer cell proliferation and apoptosis.

\section{Materials and methods}

Collection of tissue samples. This study was approved by the medical research ethics committee of Sichuan University. Written informed consent was obtained from the patients. Colorectal adenocarcinoma tissues and adjacent normal colorectal tissues were obtained from 30 colorectal adeno- 
Table I. PCR primers.

\begin{tabular}{llcc}
\hline Gene & \multicolumn{1}{c}{ Primer sequences } & Product location & Product length (bp) \\
\hline \multirow{2}{*}{ set } & Forward: 5'-GCTCAACTCCAACCACGAC-3' & $389-407$ & 120 \\
& Reverse: 5'-TCCTCACTGGCTTGTTCATTA-3' & $488-508$ & 117 \\
GAPDH & Forward: 5'-GGAAGGTGAAGGTCGGAGT-3' & $107-11$ & $205-223$ \\
& Reverse: 5'-TGAGGTCAATGAAGGGGTC-3' & 20 & \\
\hline
\end{tabular}

Table II. RT-PCR reaction.

\begin{tabular}{ll}
\hline Name & PCR Cycle \\
\hline set & $95^{\circ} \mathrm{C} 30 \mathrm{sec} ; 95^{\circ} \mathrm{C} 5 \mathrm{sec}, 62^{\circ} \mathrm{C} 30 \mathrm{sec}, 40 \mathrm{cycles} ; 95^{\circ} \mathrm{C} 15 \mathrm{sec}, 60^{\circ} \mathrm{C} 30 \mathrm{sec}, 95^{\circ} \mathrm{C} 15 \mathrm{sec}$ \\
GAPDH & $95^{\circ} \mathrm{C} 30 \mathrm{sec} ; 95^{\circ} \mathrm{C} 5 \mathrm{sec}, 60^{\circ} \mathrm{C} 30 \mathrm{sec}, 40 \mathrm{cycles} ; 95^{\circ} \mathrm{C} 15 \mathrm{sec}, 60^{\circ} \mathrm{C} 30 \mathrm{sec}, 95^{\circ} \mathrm{C} 15 \mathrm{sec}$ \\
\hline
\end{tabular}

Table III. Target sites and siRNA sequences.

\begin{tabular}{lcc}
\hline Name & Target site & siRNA sequences \\
\hline siRNA1 & GGAGGAAGGATTAGAAGAT & 5'-GGAGGAAGGAUUAGAAGAUdTdT-3' \\
siRNA2 & 3'-dTdTCCUCCUUCCUAAUCUUCUA-5' \\
& CTGGAAAGGATTTGACGAA & 5'-GGAGGAAGGAUUAGAAGAUdTdT-3' \\
siRNA3 & 3'-dTdTGACCUUUCCUAAACUGCUU-5' \\
& GATTGAACACATTGATGAA & 5'-GAUUGAACACAUUGAUGAAdTdT-3' \\
\hline
\end{tabular}

carcinoma patients at the West China Hospital of Sichuan University, China. Normal colorectal tissues were collected from the area at least $5 \mathrm{~cm}$ from the edge of the tumor. Pathological examination further confirmed no cancer cells were evident in the collected normal tissues. The tissues were snap-frozen and stored in liquid nitrogen for real-time PCR analysis.

Real-time PCR. Total RNA from tumor and matched normal tissues was isolated using TRIzol RNA isolation reagent (Invitrogen, Carlsbad, CA, USA) according to the manufacturer's protocol. Total RNA (1 $\mu \mathrm{g})$ was reverse transcribed using the M-Mulv reverse transcriptase kit (Fermentas, Ontario, Canada).

Real-time PCR was performed using SYBR Premix Ex Taq (Takara, Japan) according to the manufacturer's instruction and related international standards (6-8). cDNA $(2 \mu \mathrm{l})$ from $1 \mu \mathrm{g}$ RNA template was used for each PCR reaction. The amplification was performed on the Bio-Rad C1000 Real-Time Thermal Cycler (Bio-Rad, Philadelphia, PA, USA). GAPDH gene was used as an endogenous control. PCR primers of the set gene and GAPDH control are shown in Table I. The conditions of PCR reactions are shown in Table II.

siRNA selection. Based on the mRNA sequence of set gene, three pairs of siRNA (small interfering RNA) sequences were selected by siRNA design software on line (http://www.ambion . com). The target sites and corresponding siRNA sequences are shown in Table III. Using the BLASTn program, we confirmed that the selected siRNA sequences lacked homology to any other human coding sequences. Negative control siRNA did not cause any gene silencing, and was only used to test the specificity of the siRNA.

Human colon carcinoma cell line Ls174 (ATCC, Manassas, VA, USA) and oral squamous cell carcinoma cell line HSC3 (Tissue and Cell Culture Center, Japan), were transfected with set siRNA. Ls174 and HSC3 cells were cultured in DMEM (10\% FBS, $100 \mathrm{U} / \mathrm{ml}$ penicillin and $100 \mathrm{U} / \mathrm{ml}$ streptomycin) at $37^{\circ} \mathrm{C}$ with $5 \% \mathrm{CO}_{2}$. Each type of cell was divided into five groups: the non-treatment control group, negative control group (control siRNA), and the siRNA1, siRNA2 and siRNA3 groups. Following transfection with siRNA sequences or controls for $48 \mathrm{~h}$, total RNA was isolated from each group. After reverse transcription, the set gene expression level was examined by real-time PCR. The GAPDH gene was used as the endogenous control. The siRNA sequences, which exhibited the highest inhibition effect on set gene expression, were selected for subsequent experiments.

MTT assay. MTT assay was performed to examine the cell proliferation. Cells in the logarithmic phase were cultured in 96-well culture plates. Three groups were set up as follows: the non-treatment control, negative control (control siRNA), 
Table IV. set gene expression in colorectal adenocarcinoma and normal colorectal tissue.

\begin{tabular}{lllll}
\hline Name & Min & Max & Mean & Standard error \\
\hline Adenocarcinoma & 0.025011 & 0.47049 & 0.0996757 & 0.09177069 \\
Normal & 0.02764 & 0.26622 & 0.0697897 & 0.04925080
\end{tabular}

Table V. set gene expression change and patient characteristics such as gender, age, Dukes' stage and differentiated degree.

\begin{tabular}{|c|c|c|c|c|c|c|c|c|c|c|}
\hline \multirow[b]{2}{*}{ Expression } & \multicolumn{2}{|c|}{ Gender } & \multicolumn{2}{|c|}{ Age } & \multicolumn{3}{|c|}{ Dukes stage } & \multicolumn{3}{|c|}{ Differentiated degree } \\
\hline & Male & Female & $\geq 50$ & $<50$ & B & $\mathrm{C}$ & $\mathrm{D}$ & High & Middle & Low \\
\hline Up-regulation & 10 & 10 & 17 & 3 & 3 & 12 & 5 & 1 & 15 & 4 \\
\hline Down-regulation & 7 & 3 & 9 & 1 & 3 & 5 & 2 & 0 & 8 & 2 \\
\hline
\end{tabular}

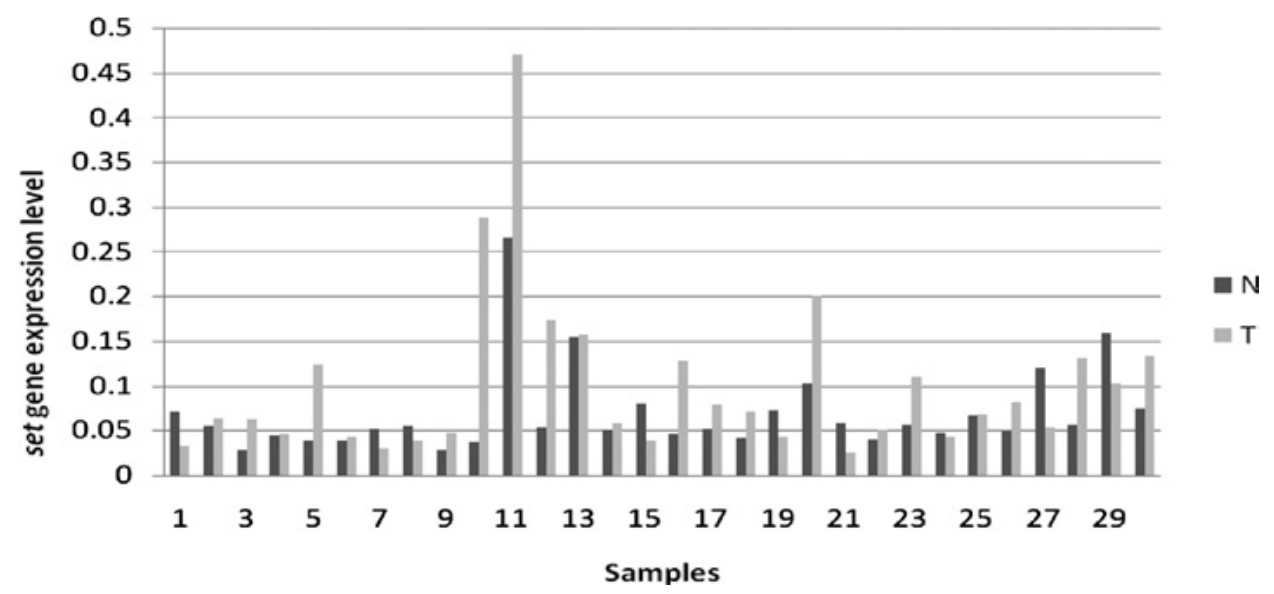

Figure 1. Expression level of the set gene ( $\mathrm{T}=135, \mathrm{P}=0.045$ by Wilcoxon signed-rank test). $\mathrm{T}$, colorectal adenocarcinoma sample; $\mathrm{N}$, matched normal sample).

and experimental groups. Each group had six replicates. MTT reagent $(20 \mu \mathrm{l})$ (Sigma, St. Louis, MO, USA) were added at each time point $(6,12,36$ and $48 \mathrm{~h})$. After the cells were cultured for a further $4 \mathrm{~h}$, the medium was removed and $150 \mu \mathrm{l}$ DMSO was added into each well, and incubated for $15 \mathrm{~min}$. The plate was read at an absorbance wavelength of $490 \mathrm{~nm}$ by a microplate reader (Bio-Rad, Model 680, USA). The experiment was repeated three times.

Flow cytometry. set siRNA effect on cell apoptosis was studied by flow cytometry (BD FACSAria II Cell Sorter, BD Co., USA). Cells in the logarithmic phase were collected and cultured in six-well culture plates. Three groups were set up as follows: the non-treatment control, negative control (control siRNA) and experimental groups. Each group had triplicates. In the cell apoptosis experiment, cells were collected $24 \mathrm{~h}$ posttransfection, using PBS to wash the cells twice (2000 rmp, $5 \mathrm{~min}, 4^{\circ} \mathrm{C}$ ). The cells were floated in $400 \mu \mathrm{l} \mathrm{PBS}, 5 \mu \mathrm{l}$ Annexin $\mathrm{V}$-FITC was added and agitated gently, and the mixture was placed for $10 \mathrm{~min}$ in room temperature. The mixture was placed into $10 \mu \mathrm{l} \mathrm{PI}(20 \mu \mathrm{g} / \mathrm{ml})$, and was incubated for $30 \mathrm{~min}$ in $4^{\circ} \mathrm{C}$, and then cell apoptosis was tested by flow cytometry. The experiment was repeated three times.
Statistical analysis. Values are given as the means \pm standard error of the mean (SEM). Statistical analysis was performed using the Student's t-test, Wilcoxon signed-rank test, Fisher's exact probabilities test, or ANOVA with SPSS17.0. P<0.05 was considered to be statistically significant.

\section{Results}

set gene expression level. To determine the set gene expression profile in human colorectal adenocarcinoma tissues, we collected 30 pairs of tumor samples and matched normal tissue samples. Real-time PCR was used to detect the mRNA level of the set gene. set gene expression was up-regulated in $70 \%$ of tumor samples ( 21 out of 30 samples). Of these, 6 samples had a fold change $>2$-fold, ranging from 2.24- to 7.9-fold, compared to the adjacent normal colorectal tissues (Table IV, Fig. 1). No significant correlation was found between set gene expression and gender, age, Dukes' stage, and differentiated degree by Fisher's exact probabilities test (Table V).

siRNA selection. To examine the effect of set siRNA on cancer cell lines, the human colon carcinoma cell line Ls174 and oral squamous cell carcinoma cell line HSC3, were transfected with 
A

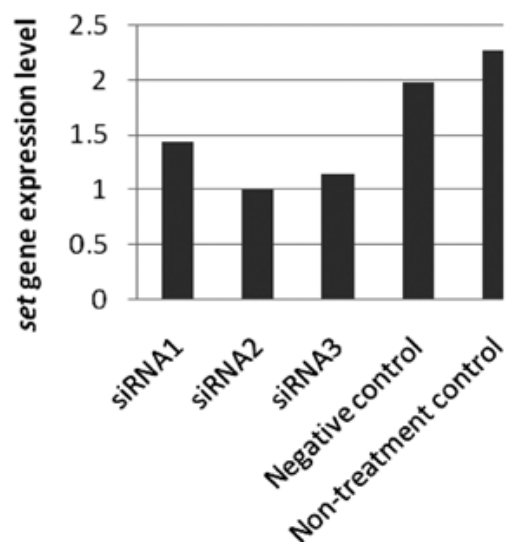

B

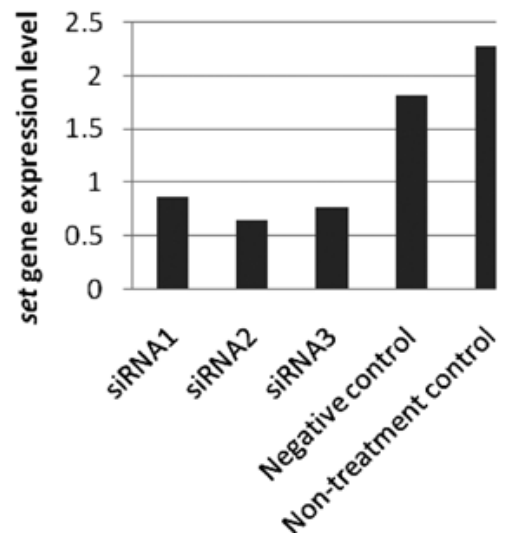

Figure 2. (A) set expression level in Ls174 cells. (siRNA inhibition of the siRNA1, siRNA2 and siRNA3 groups, and the negative control group was 36.82 , 55.91, 49.71 and $12.77 \%$, respectively). (B) set expression level in HSC3 cells. (siRNA inhibition of the siRNA1, siRNA2 and siRNA3 groups, and the negative control group was $62.38,71.57,61.16$ and $20.06 \%$, respectively).

Table VI. set gene expression in each group.

\begin{tabular}{llcccc}
\hline Groups & siRNA1 & siRNA2 & siRNA3 & Negative control & Non-treatment control \\
\hline Ls174 & $1.433 \pm 0.0453$ & $1 \pm 0.0374$ & $1.141 \pm 0.166$ & $1.978 \pm 0.041$ & $2.268 \pm 0.129$ \\
HSC3 & $0.856 \pm 0.037$ & $0.647 \pm 0.155$ & $0.77 \pm 0.038$ & $1.818 \pm 0.35$ & $2.275 \pm 0.139$ \\
\hline
\end{tabular}

Table VII. LS174 cell proliferation.

\begin{tabular}{cccccc}
\hline Time points $(\mathrm{h})$ & set siRNA & Negative control & $\begin{array}{c}\text { Non-treatment } \\
\text { control }\end{array}$ & F & P \\
\hline 6 & $0.414 \pm 0.011$ & $0.417 \pm 0.015$ & $0.452 \pm 0.026$ & 5.483 & 0.028 \\
12 & $0.455 \pm 0.021$ & $0.493 \pm 0.033$ & $0.524 \pm 0.031$ & 5.5 & 0.028 \\
24 & $0.505 \pm 0.03$ & $0.582 \pm 0.043$ & $0.633 \pm 0.032$ & 13.06 & 0.002 \\
36 & $0.674 \pm 0.035$ & $0.746 \pm 0.031$ & $0.787 \pm 0.043$ & 9.625 & 0.006 \\
48 & $0.82 \pm 0.018$ & $0.89 \pm 0.035$ & $0.909 \pm 0.04$ & 8.12 & 0.01 \\
\hline
\end{tabular}

set siRNA. Compared to the non-treatment control group, the siRNA2 group indicated a 55.91\% inhibition in Ls174 cells and $71.57 \%$ in HSC3 cells. This inhibition was greater than that of the siRNA1 and siRNA3 groups (Table VI, Fig. 2). Therefore, siRNA2 sequences were used for subsequent experiments.

Cell proliferation. Using the selected siRNA sequences against the set gene, we examined its role in cell proliferation in the Ls174 and HSC3 cells. MTT assay showed that after interference for $24 \mathrm{~h}$, cell proliferation of both Ls174 and HSC3 cells was significantly inhibited in the set siRNA group compared to the non-treatment control and negative control groups. The inhibition rate was $21.4 \%$ in the Ls174 cell lines (Table VII), and $20.2 \%$ in the HSC3 cell lines (Table VIII). This result suggested that siRNA inhibition of the set gene suppressed cancer cell proliferation.
Cell apoptosis. To examine the role of the set gene in cell apoptosis, we performed flow cytometry in Ls174 and HSC3 cells transfected with the selected siRNA sequences against the set gene. After transfection for $24 \mathrm{~h}$, the cell apoptosis rate of the set siRNA, negative siRNA control and non-treatment control groups in Ls174 cells was 18.37 $\pm 2.97,12.23 \pm 0.87$ and $11.32 \pm 1.15 \%$ (Fig. 3A). The cell apoptosis rate of the set siRNA group was significantly higher than that of the negative siRNA control $(\mathrm{t}=-4.854, \mathrm{p}=0.003)$ and non-treatment control groups $(\mathrm{t}=-5.421, \mathrm{p}=0.001)$. No statistical significance was noted between the negative siRNA control and non-treatment control groups $(\mathrm{t}=-1.552, \mathrm{p}=0.154)$. The apoptosis rate of the set siRNA, negative siRNA control and non-treatment control groups in HSC3 cells was 17.97 $\pm 2.77,8.53 \pm 0.91$ and $7.85 \pm 0.62 \%$, respectively (Fig. 3B). The cell apoptosis rate of the set siRNA group was significantly higher than that of 
Table VIII. HCS3 cell proliferation.

\begin{tabular}{rrrrr}
\hline Time points $(\mathrm{h})$ & set siRNA & Negative control & $\begin{array}{c}\text { Non-treatment } \\
\text { control }\end{array}$ & $F \quad$ P \\
\hline 6 & $0.394 \pm 0.017$ & $0.395 \pm 0.018$ & $0.461 \pm 0.023$ & 15.913 \\
12 & $0.437 \pm 0.029$ & $0.477 \pm 0.033$ & $0.536 \pm 0.031$ & 10.350 \\
24 & $0.493 \pm 0.039$ & $0.574 \pm 0.035$ & $0.627 \pm 0.034$ & 13.820 \\
36 & $0.7 \pm 0.046$ & $0.803 \pm 0.054$ & $0.816 \pm 0.038$ & 7.429 \\
48 & $0.83 \pm 0.045$ & $0.941 \pm 0.041$ & $0.953 \pm 0.033$ & 0.005 \\
\hline
\end{tabular}

A
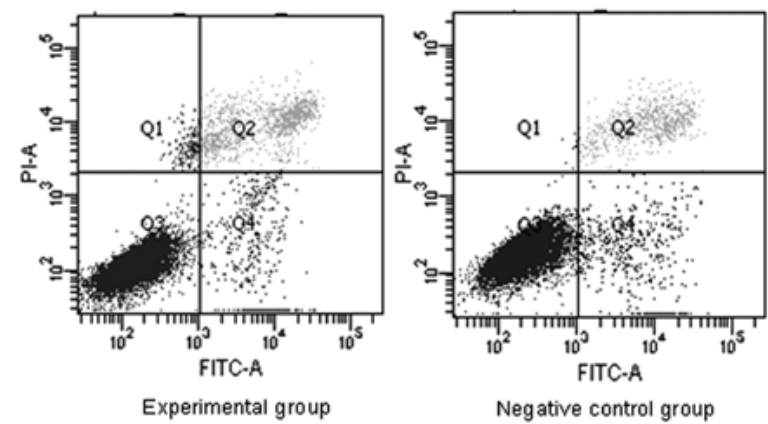

B

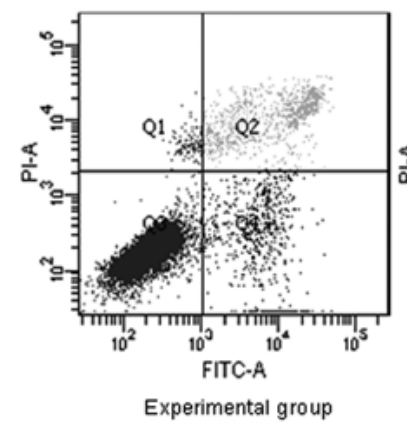

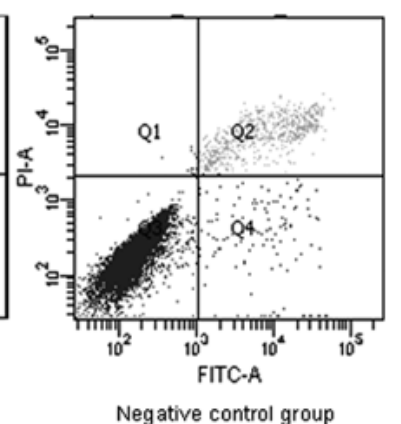

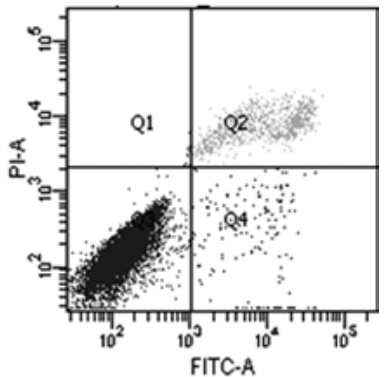

Non-tre atment control group

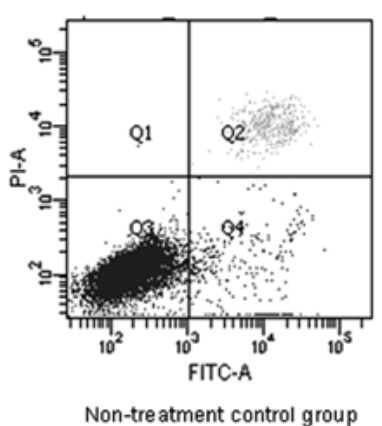

Figure 3. (A) Cell apoptosis of Ls174 and (B) HSC3 cells.

the negative siRNA control $(\mathrm{t}=7.916, \mathrm{p}=0)$ and non-treatment control groups $(\mathrm{t}=8.721, \mathrm{p}=0)$. No statistical significance was found between the negative siRNA control and non-treatment control groups $(\mathrm{t}=1.524, \mathrm{p}=0.163)$.

\section{Discussion}

In 1992, von Lindern et al (9) first identified the set gene in a patient with acute undifferentiated leukemia. Translocation of chromosome 9 resulted in the generation of the SET-CAN fusion protein. To study the role of the set gene in acute undifferentiated leukemia, Adachi et al $(10,11)$ identified the SET protein by immunoprecipitation using rabbit antiserum, and observed that this nuclear phosphoprotein was expressed ubiquitously in various human cell lines. These authors proposed that SET played a key role in the mechanism of leukemo-genesis in acute undifferentiated leukemia, possibly by activating $\mathrm{CAN}$ in nuclei and stimulating the transformation potential of SET-CAN. Saito et al (12) developed a transgenic mice strain-expressing SET-CAN protein. This transgenic mouse showed the characteristics of anemia, thrombocytopenia, and splenomegaly, with a great quantity of blood cells in peripheral blood. These symptoms were in line with those of acute undifferentiated leukemia. Furthermore, van Vlierberghe et al (13) reported set-can gene fusion in T-cell acute lymphoblastic leukemia. These authors found that the fusion protein appeared to promote an elevated expression of the HOXA cluster genes (homeobox A, HOXA), which are key genes involved in hematopoietic stem cell proliferation and differentiation, that may cause leukemia, such as T-cell acute lymphoblastic leukemia. After screening 141 human leukemia and lymphoma cell lines, Quentmeier et al (14) found that only the T-cell acute lymphoblastic leukemia cell line Loucy and the acute myeloid leukemia cell line Megal expressed the set-can fusion gene transcript. In addition to leukemia, the set gene was also found to be correlated to human ovarian cancer. In their study, Ouellet et al found that the SET complex was highly expressed in invasive grade 3 tumors in human ovarian cancer by immunohistochemistry. Notabley, overexpression of this complex was correlated with tumor differentiation (15). These studies indicate that set gene is involved in the tumorigenesis of multiple malignant tumors. However, the role of the set gene in colorectal adenocarcinoma and oral squamous cell carcinoma has yet to be determined. 
Using real-time PCR, our results showed that set gene expression was significantly up-regulated in human colorectal adenocarcinoma tissues, compared to adjacent normal colorectal tissues. Although we did not observe any significant correlation between set gene expression and patient characteristics such as gender, age, Dukes' stage, or tumor differentiation a higher rate was noted in Dukes' C $(70.59 \%)$ and D stages $(71.43 \%)$ as compared to Dukes' B stage (50\%). This tendency was in agreement with Ouellet's finding in human ovarian cancer (16). Our findings suggest that the set gene plays a role in cancer cell invasion in human colorectal adenocarcinoma. We hypothesize that set is a diagnostic marker for prognosis, particularly neoplasm invasiveness.

Our real-time PCR data indicated that set gene may be involved in the development of colorectal adenocarcinoma. To examine the role of set in the tumorigenesis of colorectal adenocarcinoma, we performed a set siRNA interference experiment to determine the effect of set inhibition on cancer cell proliferation and apoptosis. We selected human colon carcinoma cell line Ls174 and oral squamous cell carcinoma cell line HSC3 to perform these experiments.

In our study, three pairs of siRNA sequences were synthesized as candidates for RNAi. siRNA2 sequences demonstrated the highest inhibition on set gene expression in Ls174 and HSC3 cells, and were selected for the present study. MTT assay and flow cytometry results suggest that the interfering set gene inhibited cell proliferation while promoting cell apoptosis in colorectal adenocarcinoma and oral squamous cell carcinoma cell lines.

It appears that set has a different mechanism of action in tumors. Scientists (16-19) have reported that set is capable of inhibiting the acetylation of histone and regulating transcription. On the other hand, investigators (20-23) have also reported that SET regulates the process of cell division, including promoting DNA replication in adenovirus, regulating nucleosome assembly, chromosome resolution and promoting gene transcription as molecular chaperones of histone. Moreover, SET is capable of inhibiting the anti-oncogene PP2A $(24,25)$. It has been found that set increased the activity of the $(17,20)$ splitting enzyme in CYP17 and prompted the creation of $\gamma$-interferon in natural killer cells by inhibiting PP2A. PP2A plays a crucial role in cell cycle, DNA replication, signal conduction, cell differentiation and malignant tumor transformation. Therefore, set may promote tumorigenesis by inhibiting PP2A. We hypothesized that set is a potential oncogene in colorectal adenocarcinoma and oral squamous cell carcinoma, and that during tumorigenesis, it may utilize a mechanism of inhibition of histone acetylation, or PP2A.

In conclusion, our study showed that the set gene is overexpressed in human colorectal adenocarcinoma, and that the inhibition of its gene expression effectively suppresses cell proliferation and promotes cell apoptosis. We hypothesized that the set gene is a diagnostic marker and treatment target for multiple malignant tumors. However, further studies are required to confirm this hypothesis and investigate the mechanism.

\section{Acknowledgements}

This study was supported by research funds from the Oral Disease Key Laboratory of Sichuan University (SKLODSCU
20090021) and the National Natural Science Foundation of China (81072023). The authors thank the Public Laboratory of the Second Affiliated Hospital of West China of Sichuan University for critically reading the manuscript.

\section{References}

1. Boyle P and Ferlay J: Cancer incidence and mortality in Europe, 2004. Ann Oncol 16: 481-488, 2005.

2. Zheng S and Cai SR: Colorectal cancer epidermiology and prevention study in China. Chin Ger J Clin 1 Oncol 2: 72-76, 2003.

3. Ofra B, Ofer F, Avner A, et al: An orally delivered small-molecule formulation with antiangiogenic and anticancer activity. Nat Biotechnol 26: 799-807, 2008.

4. Zhang $\mathrm{C}$ and Chen Y: Electronic cloning and validating of the suppression subtractive hybridization EST ES274070 of human colorectal adenocarcinoma. US Chinese J Lymph Oncol 6: 83-88, 2007.

5. Chen Y, Zhang Y, Zhou Z, Wang G and Yi Z: Identification of differentially expressed genes in human colorectal adenocarcinoma. World J Gastroenterol 12: 1025-1032, 2006.

6. Bustin SA, Benes V, Garson JA, et al: The MIQE guidelines: minimum information for publication of quantitative real-time PCR experiments. Clin Chem 55: 4611-4622, 2009.

7. Nolan T, Hands RE and Bustin SA: Quantification of mRNA using real-time RT-PCR. Nat Protoc 1: 1560-1581, 2006.

8. Pfaffl MW: A new mathematical model for relative quantification in real-time RT-PCR. Nucleic Acids Res 29: 2002-2007, 2001.

9. Von Lindern M, Van Baal S, Wiegant J, Raap A, Hagemeijer A and Grosveld G: Can, a putative oncogene associated with myeloid leukemogenesis, may be activated by fusion of its $3^{\prime}$ half to different genes: characterization of the set gene. Mol Cell Biol 2: 3346-3355, 1992.

10. Adachi Y, Pavlakis GN and Copeland TD: Identification and characterization of SET, a nuclear phosphorprotein encoded by the translocation break point in acute undifferentiated leukemia. J Biol Chem 269: 2258-2262, 1994.

11. Adachi Y, Pavlakis GN and Copeland TD: Identification of in vivo phosphorylation sites of SET, a nuclear phosphoprotein encoded by the translocation breakpoint in acute undifferentiated leukemia. FEBS Lett 340: 231-235, 1994.

12. Saito S, Nouno K, Shimizu R, Yamamoto M and Nagata K: Impairment of erythroid and megakaryocytic differentiation by a leukemia-associated and $\mathrm{t}(9 ; 9)$-derived fusion gene product, SET/TAF-Ibeta-CAN/Nup214. J Cell Physiol 214: 322-333, 2008.

13. Van Vlierberghe $\mathrm{P}$, van Grotel M, Tchinda J, et al: The recurrent SET-NUP214 fusion as a new HOXA activation mechanism in pediatric T-cell acute lymphoblastic leukemia. Blood 111: 4668-4680, 2008.

14. Quentmeier H, Schneider B, Röhrs S, Romani J, Zaborski M, Macleod RA and Drexler HG: SET-NUP214 fusion in acute myeloid leukemia- and T-cell acute lymphoblastic leukemiaderived cell lines. J Hematol Oncol 2:3, 2009.

15. Ouellet V, Le Page C, Guyot MC, LuSSier C, Tonin PN, Proveneher DM and Mes-Masson AM: SET ComPlex in serouse Pithelial ovarian cancer. Int J Cancer 119: 2119-2126, 2006.

16. Seo SB, McNamara P, Heo S, et al: Regulation of histone acetylation and transcription by INHAT, a human cellular complex containing the set oncoprotein. Cell 104: 119-130, 2001.

17. Cervoni N, Derich N, Seo SB, et al: The oncoprotein Set/ TAF-lbeta, an inhibitor of histone acetyhetransferase inhibits active de methylation of DNA, integrating DNA methylation and transcriptional silencing. J Biol Chem 277: 25026-25031, 2002.

18. Wagner S, Weber S, Kleinschmidt MA, Nagata K and Bauer UM: SET-mediated promoter hypoacetylation is a prerequisite for coactivation of the estrogen-responsive $\mathrm{pS} 2$ gene by PRMT1. J Biol Chem 281: 27242-27250, 2006.

19. Ichijo T, Chrousos GP and Kino T: Activated glucocorticoid receptor interacts with the INHAT component Set/TAF-Ibeta and releases it from a glucocorticoid-responsive gene promoter, relieving repression: implications for the pathogenesis of glucocorticoid resistance in acute undifferentiated leukemia with Set-Can translocation. Mol Cell Endocrinol 283: 19-31, 2008.

20. Nagata K, Kawase H, Handa H, et al: Replication factor encoded by a putative oncogene, set, associated with myeloid leukemogenesis. Proc Natl Acad Sci USA 92: 4279-4283, 1995. 
21. Pegoraro G, Maroello A, Myers MP, et al: Regulation of adenoassociated virus DNA replication by the cellular TAF-I/set complex. J Virol 80: 6855-6864, 2006.

22. Chowdhury D, Beresford PJ, Zhu P, et al: The exonuclease TREX1 is in the SET complex and acts in concert with NM23-H 1 to degrade DNA during granzyme A-mediated cell death. Mol Cell 23: 133-142, 2006.

23. Zhao T, Zhang H, Guo Y, et al: Granzyme K cleaves the nucleosome assembly protein SET to induce single-stranded DNA nicks of target cells. Cell Death Differ 14: 489-499, 2007.
24. Lin M, Makkinje A and Damuni Z: The myeloid leukemia-associated protein SET is a potent inhibitor of protein phosphatase 2A. J Biol Chem 271: 11059-11062, 1996.

25. Trotta R, Ciarlariello D, Dal Col J, et al: The PP2A inhibitor SET regulates natural killer cell IFN-gamma production. J Exp Med 204: 2397-2405, 2007. 